\title{
Monitoring the Exocytosis and Full Fusion of Insulin Granules in Pancreatic Islet Cells via Graphene Liquid Cell-Transmission Electron Microscopy
}

\author{
Emre Firlar ${ }^{1,2}$, Shayan Shafiee ${ }^{1}$, Meagan Ouy ${ }^{1}$, Yuan Xing ${ }^{1,3}$, Daniel Lee ${ }^{1,3}$, Alessandro Chan ${ }^{1,3}$, \\ Solomon Afelik ${ }^{3}$, Reza Shahbazian Yassar $^{2}$,Yong Wang ${ }^{3}$, Jose Oberholzer ${ }^{1,3}$, Tolou Shokuhfar ${ }^{1,4}$ \\ 1. University of Illinois at Chicago, Department of Bioengineering, Chicago IL, USA \\ 2. University of Illinois at Chicago, Department of Mechanical and Industrial Engineering, Chicago IL, \\ USA \\ 3. University of Illinois at Chicago, Department of Surgery, Chicago IL, USA \\ 4. University of Illinois at Chicago, College of Dentistry, Chicago IL, USA
}

Investigation of sub-cellular level activities has been of a great interest for the biological, medical and materials scientists for many years. Earlier approaches to monitor the live beta cell insulin granule trafficking fusion and exocytosis remained not fully accurate due to the sample preparation and imaging techniques used. With the electron microscopy techniques used till now, samples were either fixed with chemicals, stained, embedded and sectioned [1] or cryogenically fixed and imaged [2]. Liquid cell flow holder Transmission Electron Microscopy (TEM) imaging was also proposed but it had very high thickness, which reduced the imaging and chemical characterization resolution [3]. To that end, our approach for the investigation of live cell activities is to use Graphene Liquid Cells (GLC) in TEM at $80 \mathrm{kV}$ [4]. Encapsulating the liquid media in GLC helps to obtain high resolution in both TEM and Scanning Transmission Electron Microscopy (STEM) imaging. Via this proposed technique, we were able to visualize the effects of stimulators on insulin granule size, motion, exocytosis and trafficking. We have successfully imaged and recoded the sub-cellular phenomena in real-time as shown in Fig. 1: Dynamic full fusion, Fig. 2: Sequential Fusion events under $30 \mathrm{mM} \mathrm{KCI}$ and Fig 3: Exocytosis of MIN6 cells stimulated by $30 \mathrm{mM}$ KCI. These were rarely ever observed by other conventional methodologies and will help generate novel drug development for the Diabetes treatment by comparing the healthy and pathological cells [5].

\section{References:}

[1] Nagayama, K. \& Danev, R. Philosophical Transactions of the Royal Society B: Biological Sciences 363 (2008), p. 2153.

[2] Mandelkow, E. M., Mandelkow, E. \& Milligan, R. A. Trends in Cell Biology 1 (1991), p. 153

[3] Evans, J. E. et al, Nano Letters 11 (2011), p. 2809.

[4] Wang, C. et al, Advanced Materials 26, p. 3410.

[5] The authors are grateful to the National Science Foundation, CAREER award DMR-1564950 for providing partial financial support. "This work made use of instruments in the Electron Microscopy Service (Research Resources Center, UIC)” 

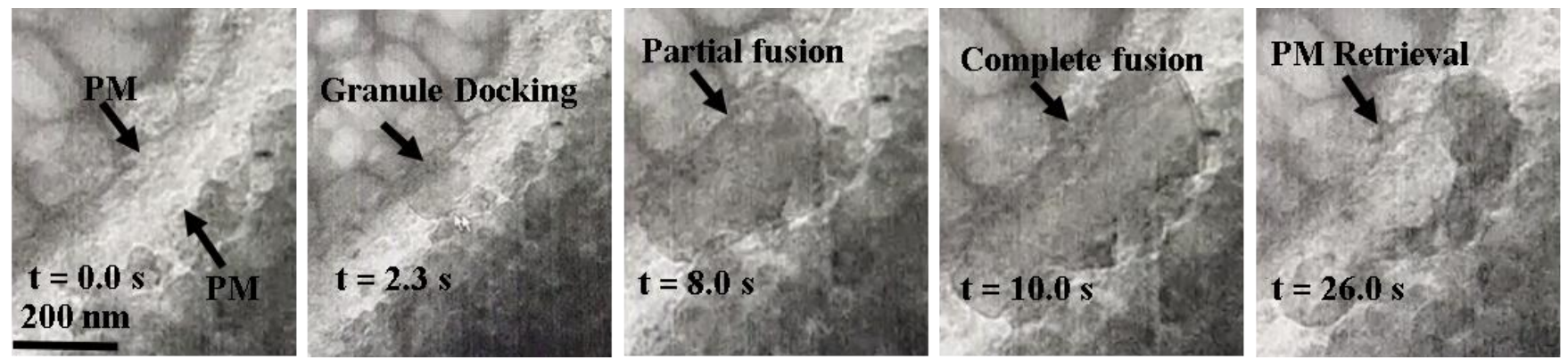

Figure 1. Time resolved images showing the insulin granule expelled from the plasma membrane, docked on the plasma membrane and following complete fusion

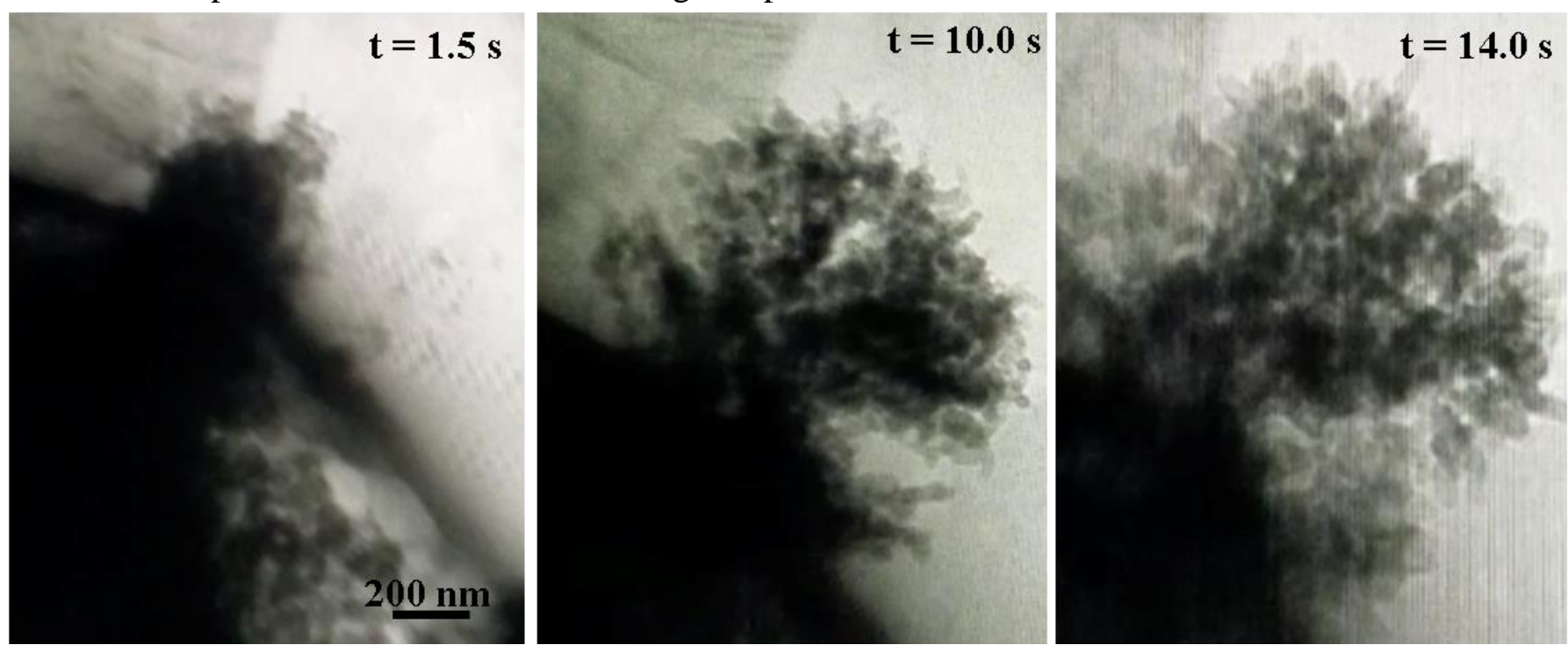

Figure 2. Time resolved images showing the sequential fusion showing first docking of insulin granule on the plasma membrane and following attachment of granules on the top of each other.

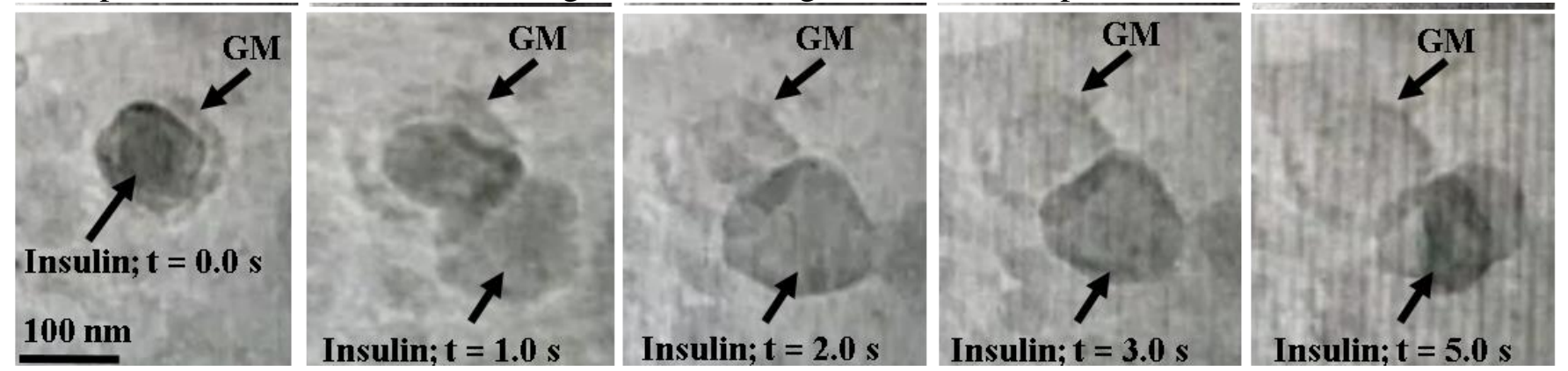

Figure 3. Time resolved images of insulin release from granules. 